\title{
Development of Simulator for LNG Carrier Liquid Cargo Handling
}

\author{
X.K. Zhang, Q. H. He \\ Key Lab. of Marine Simulation \& Control \\ Dalian Maritime University
}

\author{
J.H. Cao \\ Key Lab. of Marine Simu lation\&Control \\ Dalian Maritime University \\ Department of Computer Science and Technology \\ Dalian Neusoft University of Information
}

\begin{abstract}
To solve the difficulties in training on ship, a new simulator was developed by importing, digesting and recreating the Kongsberg simulator. With model-based and object-oriented design following by modular programming method, the whole set of system is developed by VB under Windows platforms and Sequence Query Language (SQL) Server 2008. It has functions of liquid cargo handling simulation training, training results evaluation, operation management, real-time monitoring, and simulation data playback. More than 20 kinds of mathematical model were established, including the model of the temperature, pressure, precooling, liquid level, pump, liquid cargo tank and so on. Through the experimental test, the training of loading and unloading simulation operation has been completely realized and the result has successfully fulfilled practical requirements. The work laid the foundation of the follow-up improvement and development for LNG carrier liquid cargo handling simulator.
\end{abstract}

Keywords-LNG; simulator; model; object-oriented development; training

\section{INTRODUCTION}

With the diminishing of traditional fossil energy such as oil and coal in the volume of proved reserves, natural gas as an alternative fuel is playing an important role in the energy market. Natural gas composed mostly of methane is the cleanest burning fossil fuel and has the ability to reduce the environment impact [1]. Liquefied Nature Gas (LNG) is natural gas in liquid state at atmospheric pressure and temperature around minus $161^{\circ} \mathrm{C}[2,3]$. It produces fewer pollutants than oil or coal combustion and has wide-ranging uses in industry, residential and business premises, as well as electrical power generation [4]. Proper training of ship crews for LNG carrier is vital to avoid costly mistakes during liquid cargo transfer operations. However, experienced crews are not enough according to the development of LNG industry [5]. It is hard to provide the training for the crews on board. Since the operations deal with explosive substances, making a small mistake is likely to have a big accident [6]. Thus, the training job on simulator of LNG carrier liquid cargo handling is considered as one of efficient way for training qualified personnel of LNG carrier by simulator. This fashion has been well accepted by international convention STCW-95. By this means crews can perform their functions safely and efficiently [7].

Especially, there are many mature marine simulator companies in other countries such as SSPA of Sweden,
MARIN of Holland, CAORF of American KingPoint, Kongsberg of Norway, Transas of England, Ship Analytics of U.S., Force Technology of Denmark and so on[8]. Among these products, the LNG carrier cargo handling simulators of Kongsberg and Transas are outstanding. Domestic LNG simulators are still on simulating and developing state. And these systems are independent and cannot work together. Furthermore, these simulators cannot meet the demands of individualized training needs. Thus, the work of [9] puts forward that simu lators for special operation trainings, such as for LNG ship, should be developed and related research on remote training and e-learning should be carried out as soon as possible. Therefore, the new LNG carrier cargo handling simulator is being developed with functions which can meet the needs above.

\section{MODEL-BASED DEVELOPMENT PROCESS OF THE SIMULATOR}

Before developing, the whole process of the LNG liquid cargo operations has been studied first and requirements for the simulator have been analyzed including hardware and software. According to the requirements, the architecture of simulator is constructed which is illustrated later. The devices of the ship should be modelled respectively, such as tanks, vaporizers, heaters, compressors, pumps, valves, fans, heat exchanger, pipelines and so on. All of the device models jo int each other to create a complete LNG carrier model. According to [10], a mathematical model can produce two type of information: knowledge of the system being modelled and data observations from a system's inputs and outputs. These models are converted into simulation model which can be realized in the computer. Operating data of LNG carrier liquid cargo handling process can be collected from these system models before being analysed according to [11]. When problem emerges, the process will be recycled. General process of development is illustrated in the figure 1 .

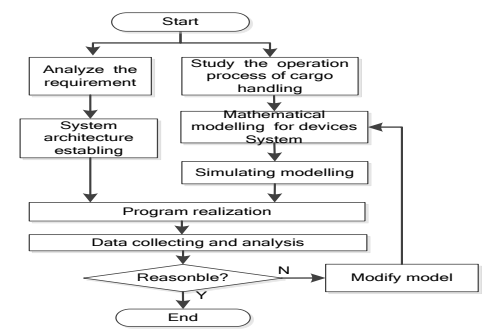

FIGURE I. DEVELOPMENT PROCESS 


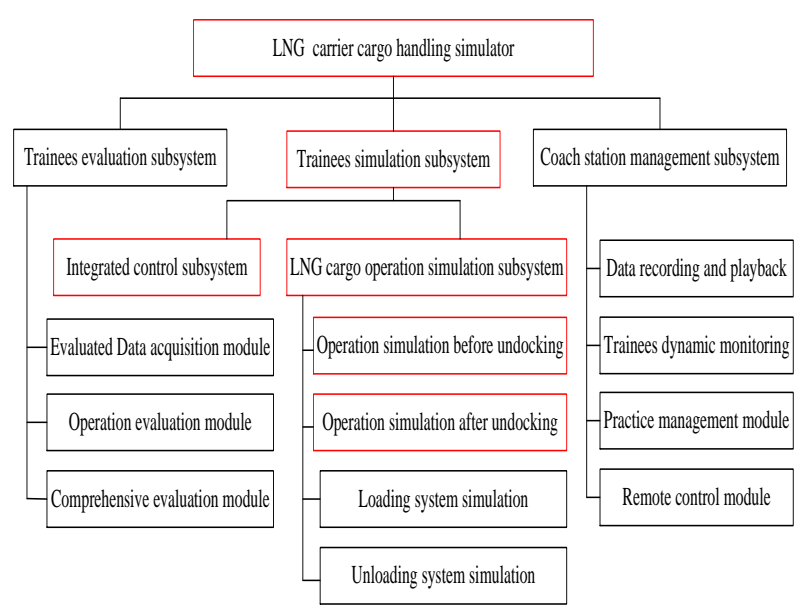

FIGURE II. SIMULATOR FUNCTIONAL CONFIGURATION.

\section{MODUlES OF LIQUID CARGO HANDLING SIMULATOR}

The simulator of liquid cargo handling for LNG carrier is consists of main four subsystem, namely trainees evaluation subsystem, trainees simulation subsystem, coach station management subsystem, integrated control subsystem. Trainees evaluation subsystem is responsible for providing the results of the trainees' simulation operation by calculating model which refers to [7]. Trainees simulation subsystem is responsible for training simulation operation. There are 13 process of LNG Carrier cargo operation refers to [5, 12-14]. Here, they are classified into four parts: operation before undocking, operation after undocking, loading operation and unloading operation. Unloading and loading simulation are already realized in this simulator. The integrated control system is responsible for the control of pressure, temperature and liquid level through hydraulic power unit, the correction of heel and trim, and high liquid level overflow alarm. Coach station management subsystem is responsible for sending exercises, editing exercises, supervising simulation operation, playback data of simulation operation, and remote control to all the workstation. The functional configuration of the simulator is shown in the figure 2 .

\section{THE SIMULATOR SySTEM ARCHITECTURE}

The workstation platform of the simulator for LNG carrier liquid cargo handling consists of industrial process control computers (IPCs) and stand-alone personal computers (PCs) which are interconnected by switch on fast Ethernet local area network. Large screens are used for demonstrating. An instructor station is used to control and monitor the state of operators' working and manage the whole training process. The user datagram protocol (UDP) and transmission control protocol / Internet protocol (TCP/IP) ensure the communication between instructor station and operating workstation. TCP Protocol is connection-oriented. Once a connection is established, transmission is bidirectional. Multiple messages are sent as packets in chunks using UDP. The instructor station also has the functions of remote-poweroff, sending exercises, exercises state monitoring and wakeon-LAN. All of data calculated by simulation model are sent to database server. The scheme of this architecture is shown in the figure 3 .

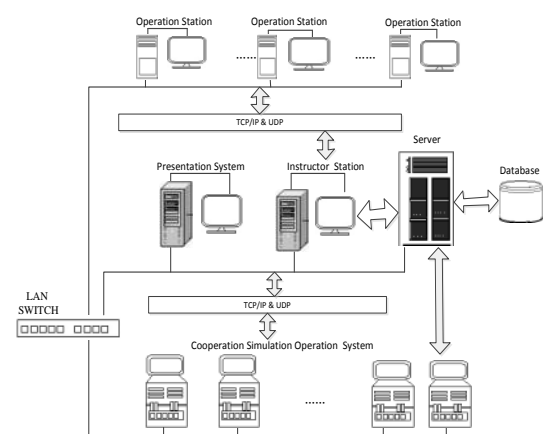

FIGURE III. SY STEM ARCHITECTURE.

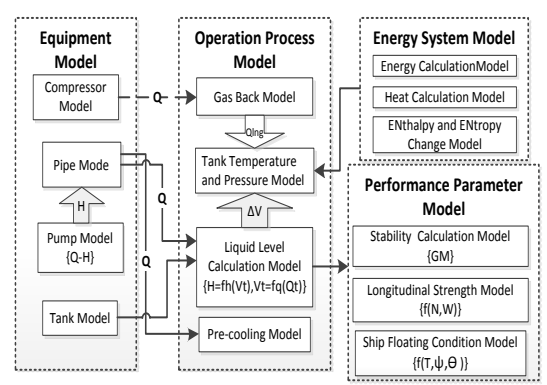

FIGURE IV. RELATIONSHIP BETWEEN MODELS.

IPCs can work in two ways, namely operation independently and cooperation. The workstation has the ability to deal with multi-task parallel processing for many concurrent operations, such as human-computer interaction, data processing, network communication and so on. During software design and realization stage, event-driven mechanism is adopted to finish task scheduling. The instructor station is responsible for communicating with workstation over TCP/IP, initializing the data of cargo handling and original state, sending the exerc is es to operation station. It also can load data from database server to playback in order to ensure the correctness of simulation. Trainer can assess the simulation operation results from the data and give an appropriate score according to the evaluation algorithm by the assessment system. The evaluation work referred to [7].

\section{MATHEMATICAL MODELS}

Using models to design complex system is common method in traditional engineering disciplines. No one would imagine constructing a complex system without first constructing a variety of specialized system models. These mathematic models include four categories: equipment model, operation process model, energy system model, performance parameter model. The equipment model and energy system model provide operation process model with state parameters, and operation process model provides performance parameter model with state parameters. Through the analys is of work [12, 15-18], this paper classifies these model and presents the relationship between these several main models which is shown in the figure 4.

\section{A. Tank Model}

The main purpose of the cargo tank model is to calculate liquid level for temperature and pressure model which is to keep the temperature and pressure maintaining the adequate 
range. There are two main types of LNG ship tank system: me mb rane-type ship and self-supporting-type.

\section{B. Pump Model}

The cargo pump of carrier is usually designed by given corporation. The performance of the pump can influence the discharge flow in the pipe. The pressure head and quantity flow character can be expressed as in the work of $[13,17]$.

\section{Liquid Level Calculating Model}

According to the parameters given in the work of [14], the equation of level calculation is shown in the formula (1). In the formula (1), $h$ represents the liquid level which can be calculated by tank model. $Q$ represents the discharge flow in unit time. It can be given by pipe model through pump model indirectly. $V_{L N G_{0}}$ represents volume before discharge. $V_{L N G}$ represents volume after discharge. The result is used to calculate stability, strength and floating in the performance parameter model.

$$
\left\{\begin{array}{c}
V_{L N G}=V_{L N G_{0}}+\int_{0}^{t} Q \mathrm{dt} \\
h=f\left(V_{L N G}\right)
\end{array}\right.
$$

\section{Tank Pressure and Temperature Calculating Model}

In work of [15], the responding model was built. With this kind of model, there are four inputs and two outputs. The formula (2) is represented by transfer function.

$$
\left[\begin{array}{l}
y_{T_{0}} \\
y_{p_{0}}
\end{array}\right]=\left[\begin{array}{l}
y_{T}-C_{T} \\
y_{p}-C_{p}
\end{array}\right]=\left[\begin{array}{cccc}
\frac{K_{1}}{T_{T} s+1} & \frac{K_{2}}{T_{T} s+1} & \frac{K_{3}}{T_{T} s+1} & \frac{K_{4}}{T_{T} s+1} \\
\frac{K_{5}}{T_{p} s+1} & \frac{K_{6}}{T_{p} s+1} & \frac{K_{7}}{T_{p} s+1} & \frac{K_{8}}{T_{p} s+1}
\end{array}\right]\left[\begin{array}{c}
x_{1} \\
x_{2} \\
x_{3} \\
x_{4}
\end{array}\right]
$$

In the formula (2), the four inputs: $x_{1}$ is the discharge rate, $x_{2}$ is the gas-return speed, $x_{3}$ is the temperature of return-gas, $x_{4}$ is the temperature of liquid cargo in the cargo tank. And the two outputs: $y_{T}$ is the temperature of LNG vapor, $y_{p}$ is the pressure of LNG vapor in the cargo tank. $C_{T}$ and $C_{p}$ are the offset, in order to make the desperation and pressure reach the expectation effect.The pressure and temperature also can be modeled by mechanism method in the future work and calculated with the result produced by energy system model and equipment model.

\section{E. Precooling Model}

Precooling is the process to prevent thermal shock to the primary containment system during cargo handling. It is bound up with liquid temperature and discharge of LNG through the pipe model. In work of [9], transfer function is shown in the formula (3). $Y_{T}$ represents temperature of line pipe. $U_{T}$ is the temperature of liquid. $U_{f}$ is the discharge flow.

$$
Y_{T}=\left[\begin{array}{ll}
\frac{0.81}{3600 s+1} & \frac{0.19}{3600 s+1}
\end{array}\right]\left[\begin{array}{c}
U_{T} \\
U_{f}
\end{array}\right]
$$

\section{F. Performance Parameter Models}

The performance parameter model is based on ship theory. All the operation of the liquid cargo handling will has an effect on this model. The ship floating condition model can be achieved by liquid cargo quality distribution in the tanks. The stability calculation model is set up by ship parameters and parameters produced from the operation process model. Longitudinal strength model is set up by the integral of distribution curve of liquid cargo weight.

\section{RESUlT OF CARGO HANDLING SIMULATION}

In order to achieve the mathe matical model of LNG carrier, a special prototype ship is chosen as the simulation object to be studied and modelled. Take a special LNG carrier for example. The specification of the ship is shown in the table 1.

TABLE I. GENERAL SPECIFICATION OFA LNGCARRIER.

\begin{tabular}{|c|c|c|c|c|}
\hline Cargo Tank & $\begin{array}{c}\text { Capacity } \\
\text { (rated flow) }\end{array}$ & LOA & B readth & $\begin{array}{c}\text { Deadweight } \\
\text { of Summermark }\end{array}$ \\
\hline $\begin{array}{l}144291.4 \mathrm{~m}^{3} \\
98 \% \text { loading }\end{array}$ & $1,500 \mathrm{~m}^{3} / \mathrm{h}$ & $291.50 \mathrm{~m}$ & $43.35 \mathrm{~m}$ & 83049.8 tonnage \\
\hline
\end{tabular}

With these models above, the simulator is being developed by VB under Windows platform with SQL Server 2008. Object-oriented method and modular programming method have been taken into considered during the development. Take an unloading simulation process as example, Fig. 5 show the curves of pressure, temperature and liquid level during unloading simulation process. Whole process is conducted according to the ratio of 1:50 and takes 14.2 minutes which equals 11.8 hours actually.

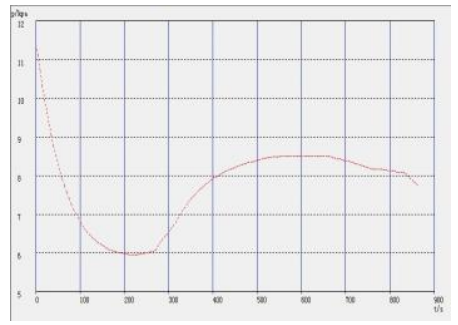

(a)

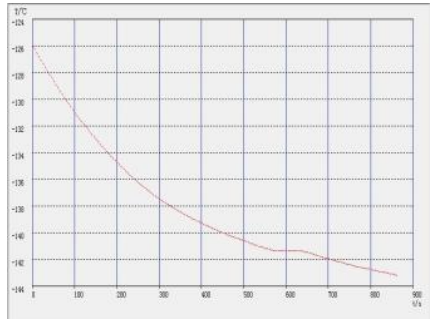

(b)

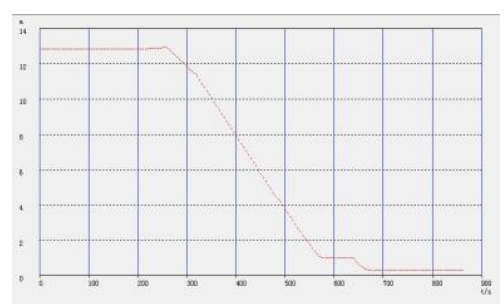

(c)

FIGURE V. PRESSURE(A), TEMPERATURE(B) AND LIQUID LEVEL(C) OF UNLOADING HANDLING.

It can be seen from the figures, the initial phase of pressure will drop rapidly. When it drops to about 6kpa, the pressure begins to rise and is finally levelling out at around 8-10kpa. Temperature slows down to keep below $-140{ }^{\circ} \mathrm{C}$ and liquid levels keeps slows down until liquid is unloading completely.

\section{CONCLUSIONS}

The new simulator discussed in this paper applied the technology of database, network, object-oriented and modular programming. LNG carrier liquid cargo simulation operation of loading and unloading are fully realized. All the subsystems 
are integrated into a whole set of system. A special LNG carrier is taken for example to carried out the mathematic models which are transformed into simulation models. Aiming at the key problems in developing, the relationship among all the main models is investigated. The simulator has modified the logic error in Kongsberg simulator. Automatic evaluation subsystem is also added to the simulator. Through the experimental test, the system runs steadily and reliability. The functions of alarm and data display are normal and test result is satisfying. The next work is to optimize the mathematic models, realize control algorithm, create $3 \mathrm{D}$ views, and integrate the correction of heel and trim. We hope that researchers can enhance and further their research work through this paper.

\section{ACKNOWLEDGMENT}

This work is partially supported by National Natural Science Fund of China (Grant No.51109020), and the Programme of Introducing Talents of Discipline to Universities (Grant No. B08046), and the Fundamental Research Funds for the Central Universities (Grant No. 3132014302).

The authors also gratefully acknowledge the helpful comments and suggestions of the reviewers, which have improved the presentation.

\section{REFERENCES}

[1] Zoolfakar M.R., Mesbahi E. and Norman R., Impact Analysis of LNG carrier Modelling systems, IEEE Symposium on Business, Engineering and Industrial Applications (ISBEIA), Langkawi, Malaysia, pp. 34-39, 2011.

[2] Luis Castillo and Carlos A. Dorao, Consensual decision-making model based on game theory for LNG processes, Energy Conversion and Management, vol.64, pp.387-396, 2012.

[3] SIGTTO. LNG Shipping Knowledge,A Witherby Seamanship Publication, Witherby Seamanship International Ltd, Livingston, United Kingdom, 2008.

[4] M.J.Ivings, C.J.Lea, D.M.Webber, et al., A protocol for the evaluation of LNG vapour dispersion models, Journal of Loss Prevention in the Process Industries, 26(1), pp. 153-163, 2013.

[5] Q.H. He, W.J. Zhang and J. Zhao, Application of LNG Cargo Operation Simulator in the Sailor Training, Marine Education Research, 29(4), pp.59-61, 2012.

[6] Jae Ik Lee, Won Jae Choi and Ga Ram Lim, Development of 270k cbm LNG- FSRU Operator Training System, Proceedings of the Twentysecond International Offshore and Polar Engineering Conference, Rhodes, Greece, pp.889-894, 2012.

[7] X.K. Zhang, Z.Z. Yang and H.Q. Hao. The Evaluation System of LNG Liquid Cargo Operations Simulator Training, Journal of Dalian Maritime University, 39(4), pp. 13-16, 2013.

[8] G.Q. Zhang, X.K. Zhang and Y.C. Jin, Overview of Liquid Cargo Handling Simulator of LNG Carriers, Navigation of China, 35(1), pp.4146, 2012.

[9] Y.C. Jin and Y. Yin. Development Strategy of Marine Simulat or in Light of the Manila Amendments to STCW Convention, Navigation of China, 35(3), pp.5-10, 2012.

[10] W.J.Karplus, The spectrum of mathematical modeling and system simulation, Mathematics and Computers in Simulation, 19(1), pp 3-10, 1977.

[11] Evert Lataire, Marc Vantorre, Guillaume Delefortrie, et al, Mathematical modelling offorces acting on ships during lightering operations, Ocean Engineering, vol.55, pp.101-115, 2012.

[12] M.J. Hu, Q.M. Shang, S. Su, et al., Simulation implementation of the liquid cargo operating process of LPG vessels, ship engineering, $\mathbf{3 0}(\mathbf{6})$, pp.55-58, 2008.

[13] J.Q. Hu, Simulation study of the liquefied cargo system of LNG carrier, Dalian Maritime University, 2003.

[14] J.J. Yu. Simulation study on the main liquefied cargo system of LNG carrier, Dalian Maritime University, 2008.

[15] Z.Z. Yang, Xian-Ku Zhang and Jing-Hua Cao, Discharging Part of LNG Liquid Cargo Handling Simulator, Research Journal of Applied Sciences, Engineering and Technology, 6(21), pp.3978-3985, 2013.

[16] Z.Z. Yang, Process of Discharge Simulation of Liquid Cargo Handling Simulat or of LNG Carrier, Dalian Maritime University, 2013.

[17] N. Liu, Simulation of Loading Operation of LNG Carrier Cargo Handling Simulator, Dalian Maritime University, 2013.

[18] C.L. Jiang, Development research on Coach Station of LNG Cargo Handling Simulator, Dalian Maritime University, 2013. 